\title{
Risk of bleeding after hospitalization for a serious coronary event: a retrospective cohort study with nested case-control analyses
}

Antonio González-Pérez ${ }^{1,2}$, María E. Sáez ${ }^{1,2}$, Saga Johansson ${ }^{3}$, Anders Himmelmann³ and Luis A. García Rodríguez ${ }^{\text {* }}$

\begin{abstract}
Background: Bleeding events have been associated with the use of antiplatelet agents. This study estimated the incidence of bleeding events in patients previously hospitalized for a serious coronary event and determined the risks of bleeding associated with the use of acetylsalicylic acid (ASA) and/or clopidogrel.
\end{abstract}

Methods: A UK primary care database was used to identify 27,707 patients aged 50 to 84 years, hospitalized for a serious coronary event during 2000 to 2007 and who were alive 30 days later (start date). Patients were followed up until they reached an endpoint (hemorrhagic stroke, upper or lower gastrointestinal bleeding [UGIB/LGIB]), death or end of study [June 30, 2011]) or met an exclusion criterion. Risk factors for bleeding were determined in a nested case-control analysis.

Results: Incidences of hemorrhagic stroke, UGIB, and LGIB were 5.0, 11.9, and 25.5 events per 10,000 person-years, respectively, and increased with age. UGIB and LGIB led to hospitalization in 73 and $23 \%$ of patients, respectively. Non-users of ASA, who were mostly discontinuers, and current users of ASA had similar risks of hemorrhagic stroke, UGIB, and LGIB. Users of combined antithrombotic therapy (warfarin and antiplatelets) experienced an increased risk of hemorrhagic stroke (odds ratio [OR], 6.36; $95 \%$ confidence interval [Cl], 1.34-30.16), whereas users of combined antiplatelet therapy (clopidogrel and ASA) experienced an increased risk of UGIB (OR, 2.42; $95 \% \mathrm{Cl}, 1.09-5.36)$. An increased risk of $\mathrm{LGIB}(\mathrm{OR}, 1.86 ; 95 \% \mathrm{Cl}, 1.34-2.57)$ was also observed in users of clopidogrel.

Conclusions: In patients previously hospitalized for a serious coronary event, combined antithrombotic therapy was associated with an increased risk of hemorrhagic stroke, whereas combined antiplatelet therapy was associated with an increased risk of UGIB.Non-use of ASA was rare in this population and use of ASA was not associated with a significantly increased risk of hemorrhagic stroke, UGIB, or LGIB.

Keywords: Acetylsalicylic acid, Bleeding, Clopidogrel, Coronary event, Dual antiplatelet therapy

Abbreviations: ASA, Acetylsalicylic acid; Cl, Confidence interval; HR, Hazard ratio; LGIB, Lower gastrointestinal bleeding; NSAID, Non-steroidal anti-inflammatory drug; OR, Odds ratio; OTC, Over-the-counter; PCP, Primary care physicians; PPI, Proton pump inhibitors; RR, Relative risk; THIN, The health improvement network; UGIB, Upper gastrointestinal bleeding

\footnotetext{
* Correspondence: lagarcia@ceife.es

${ }^{1}$ Spanish Centre for Pharmacoepidemiologic Research (CEIFE), Madrid, Spain

Full list of author information is available at the end of the article
} 


\section{Background}

Cardiovascular disease remains the principal cause of mortality in Europe, being responsible for over 4 million deaths each year [1]. For patients with acute coronary syndrome (ST-elevation or non-ST-elevation myocardial infarction, or unstable angina), resulting from occlusion of the coronary arteries, long-term antiplatelet therapy with low-dose acetylsalicylic acid (ASA) is recommended, with the addition of a $\mathrm{P}_{2} \mathrm{Y}_{12}$-receptor inhibitor, such as clopidogrel, for the first 12 months of treatment [2, 3]. Dual antiplatelet therapy (ASA in combination with a $\mathrm{P}_{2} \mathrm{Y}_{12}$-receptor inhibitor) is also recommended after revascularization of the coronary arteries [4]. These agents have been shown to reduce the risk of recurrent cardiovascular events significantly [5-9]. In addition to reducing cardiovascular risk, however, antiplatelet therapies confer an increased risk of bleeding in general [6-8] and of upper gastrointestinal bleeding (UGIB) in particular [10-12]. The risk of lower gastrointestinal bleeding (LGIB) associated with antiplatelet therapies, however, has rarely been studied and only in small populations [13-15] or elderly patients [16].

We have conducted a retrospective cohort study with nested case-control analyses in a large population to assess the incidences of hemorrhagic stroke, UGIB, and LGIB in patients who have survived a serious coronary event (defined as myocardial infarction, unstable angina, or revascularization of the coronary arteries). The effects of treatment with low-dose ASA and clopidogrel, alone or in combination, were evaluated, as well as the effects of other demographic factors, comorbidities, and comedications.

\section{Methods}

\section{Data source}

Data were collected from The Health Improvement Network (THIN), a computerized medical research database containing systematically recorded, anonymized data on over 3 million individuals currently registered with participating primary care practices in the UK [17]. Patients included in the database are representative of the general UK population with respect to age, sex, and geographical region [18]. Information contained in THIN includes patient demographics and details of consultations with primary care physicians (PCPs), and data on consultant referrals, hospitalizations, laboratory test results, diagnoses, and prescriptions. Read classification is used to code specific diagnoses [19] and a drug dictionary based on data from the Gemscript classification is used to code prescriptions [20]. The validity of THIN for use in pharmacoepidemiological research has been demonstrated [21].

\section{Ethics statement}

The company that owns THIN (Cegedim Strategic Data) has received ethical approval from the South East
Research Ethics Committee to supply anonymized, precollected, primary care data for scientific research. Patients can opt out of having their depersonalized records collected; therefore, patient consent is not required when working with anonymized records in THIN. This study was approved by a multicenter research ethics committee (REC reference: 09/H0305/90).

\section{Study cohort}

The selection of the study cohort has been described in detail elsewhere [22]. Briefly, patients were identified who were aged 50 to 84 years at any time between January 1, 2000 and December 31, 2007, who had also been enrolled with their PCP for at least 1 year, and who had at least 1 year of medical records available since their first recorded prescription. Patients were excluded if they had a diagnosis of cancer recorded before their start date, or if they were aged 70 years or over and had been enrolled with their PCP for more than 1 year and had fewer than two health contacts during that time (proxy for incomplete data recording). All individuals were identified who had evidence of hospitalization for a serious coronary event (defined as myocardial infarction, revascularization of the coronary arteries [elective and non-elective percutaneous coronary intervention or coronary artery bypass graft] or unstable angina) and who were alive 30 days later $(N=27,707)$. Each patient's start date was set at 30 days after the date of hospitalization for their coronary event. Although the original cohort [22] included patients with data recorded in both THIN and the Clinical Practice Research Datalink databases, for the current analysis we assessed only those with data included in THIN.

\section{Case ascertainment Hemorrhagic stroke}

To identify cases of hemorrhagic stroke, we followed up all patients in the study cohort from their start date until June 30, 2011, or until they reached one of the following endpoints: diagnosis of hemorrhagic stroke; met an exclusion criterion (diagnosis of cancer, alcohol abuse, or reached 85 years of age); death; or no further contribution from their PCP to THIN. The anonymized profiles of all patients with a Read code suggestive of hemorrhagic stroke were reviewed manually to ascertain genuine cases. The definition of hemorrhagic stroke was the same as that used in a recent population-based study [23]. Potential cases were discarded if: they had a Read code suggestive of hemorrhagic stroke, but that was not confirmed later; the stroke was ischemic rather than hemorrhagic; the episode was not a primary event (e.g. if it was secondary to trauma); the patient had experienced subdural hemorrhage; the stroke had occurred in a hospital setting; or they were prescribed an antiplatelet or oral 
anticoagulant in the 60 days following the event. For those with hemorrhagic stroke, the date of hospitalization was considered the index date.

\section{Gastrointestinal bleeding}

For the analysis of patients who had experienced UGIB or LGIB, we excluded all patients with a history of esophageal varices, Mallory-Weiss syndrome, chronic liver disease, or coagulopathies recorded before their start date. All patients free from these comorbidities were followed up from their start date until June 30, 2011, or until they reached one of the following endpoints: diagnosis of UGIB or LGIB; met an exclusion criterion (cancer, esophageal varices, Mallory-Weiss syndrome, chronic liver disease or coagulopathy diagnoses, alcohol abuse, or reached the age of 85 years); death; or no further contribution from their PCP to THIN. The anonymized profiles of all patients with a Read code suggestive of UGIB or LGIB were reviewed manually to ascertain genuine cases. At this stage, all patients who had been hospitalized for a nongastrointestinal event in the month before their UGIB or LGIB diagnosis were also excluded, as were all patients who met an exclusion criterion in the 2 months after their UGIB or LGIB diagnosis. Confirmed cases of UGIB were all individuals who had been referred to a consultant or admitted to hospital, for whom the site of bleeding or perforation was the stomach or duodenum. Patients whose site of bleeding or perforation was the esophagus were excluded. Confirmed cases of LGIB were all those who had been referred to a consultant or admitted to hospital, for whom the cause of bleeding had been identified (diverticulosis, polyposis, inflammatory bowel disease, ischemic colitis, etc.) and the site of bleeding or perforation was the jejunum, ileum, colon, or rectum. Cases of bleeding due to hemorrhoids were discarded. To validate cases of LGIB further, questionnaires were sent to the PCPs of a random sample of 124 potential LGIB cases requesting all information related to that event. This approach was previously used for ascertainment of cases of hemorrhagic stroke [23] and UGIB [24]. We received questionnaires with information for 101 LGIB cases (response rate, $81.5 \%)$. Most of these cases (83/101) were confirmed after reviewing the information sent, yielding a positive predictive value of $82.2 \%$. We could not confirm the LGIB diagnoses in the other 18 cases (hemorrhoids/ anal fissure identified as the cause of bleeding $[n=12]$ or no clear cause of bleeding identified $[n=6])$. For all patients with either UGIB or LGIB, the date of the first objective sign of the bleeding episode was considered the index date.

\section{Control selection}

For all patients in the study cohort, a random date was generated during the study period. If that random date fell within their follow-up period, they were eligible to be considered as a control and the random date was considered to be their index date. From this total set of eligible controls, three random control groups were selected, frequency matched by age (within 1 year), sex, and calendar year to the groups of patients with hemorrhagic stroke, UGIB, or LGIB. For comparison with patients with hemorrhagic stroke and UGIB, different control groups comprising 1,000 individuals were selected. For comparison with patients with LGIB, a control group comprising 2,000 individuals was selected.

\section{Categorization of drug use}

Exposure to drugs was classified as 'current' when the supply of the most recent prescription lasted until the index date or ended in the month before the index date, 'recent' when it ended between 1 month and 3 months before the index date, 'past' when it ended between 3 months and 1 year before the index date, and 'non-use' when there was no recorded use in the year before the index date. Duration of use was defined according to the time period of 'consecutive' prescriptions. Prescriptions were considered to be consecutive when the time interval between the end of the supply of the first and the beginning of supply of the second was less than 2 months. Treatment duration was categorized as no more than 1 month, 1 month to 1 year, and greater than 1 year. Only prescriptions issued after the start date were considered when estimating drug exposure. In the nested case-control analyses, use of ASA and use of clopidogrel include the use of each drug alone and in combination with each other, unless otherwise stated.

\section{Statistical analysis}

Odds ratios (ORs), $95 \%$ confidence intervals (CIs) and $P$ values (Wald tests), calculated using unconditional logistic regression models, were used to determine the association between the use of ASA or clopidogrel and the occurrence of hemorrhagic stroke, UGIB, or LGIB. Models were adjusted for frequency-matched variables (age, sex, and calendar year), length of follow-up, health services utilization (PCP visits, referrals, and hospitalizations), smoking, type of coronary event, history of peptic ulcer disease, and use of proton pump inhibitors (PPIs), ASA, clopidogrel, non-steroidal antiinflammatory drugs (NSAIDs), and warfarin. The effects of patient demographics and baseline characteristics, comorbidities, and comedications on bleeding events were also assessed. Due to the method used to select controls, ORs are unbiased estimates of rate ratios in the underlying study cohort. 


\section{Results}

Incidence of hemorrhagic stroke, LGIB, and UGIB

The study cohort comprised 27,707 individuals, with a mean age of 67.7 years (Table 1 ). There were more men than women (68.2 \% vs. $31.8 \%)$. The qualifying event was a myocardial infarction for $58.1 \%$ of patients, unstable angina for $6.9 \%$ and elective revascularization for $34.9 \%$. During follow-up, a total of 70 patients had a hemorrhagic stroke (mean follow-up: 5.0 years; standard deviation [SD]: 3.0 years), 152 experienced UGIB (mean follow-up: 4.6 years; SD: 3.0 years), and 316 experienced LGIB (mean follow-up: 4.5 years; standard deviation [SD]: 3.0 years). Among patients who had a hemorrhagic stroke, 48 experienced intracerebral hemorrhage and 22 had a subarachnoid hemorrhage. Among the 152 UGIB cases, the site of bleeding was gastric in 80 patients, duodenal in 47 , and gastroduodenal in 16 , while it was undefined in nine individuals. In total, 111 (73\%) patients with UGIB were hospitalized and distributions of bleeding sites were similar in hospitalized and nonhospitalized patients (Additional file 1). The most common causes of LGIB were diverticular disease $(n=201)$, polyps $(n=54)$, and inflammatory bowel disease $(n=49)$, accounting for $96.2 \%$ of LGIB cases, and 72 (23 \%) patients were hospitalized following LGIB. Causes of LGIB were similar in hospitalized and non-hospitalized patients (Additional file 2).
Overall, incidences of bleeding events were 5.0 (95\% CI, 3.9-6.3) per 10,000 person-years for hemorrhagic stroke, 11.9 (95 \% CI, 10.1-13.9) per 10,000 personyears for UGIB, and 25.5 (95\% CI, 22.7-28.4) per 10,000 person-years for LGIB (Fig. 1). The corresponding incidences of fatal bleeding events (death within 1 month of the bleed) were 2.2 (95\% CI, 1.5-3.1), 0.5 (95\% CI, 0.2-1.1), and 0.5 (95\% CI, 0.2-1.1) cases per 10,000 person-years, respectively. When only hospitalized patients were considered, the incidences of UGIB and LGIB were 8.7 (95\% CI, 7.1-10.4) and 5.8 (95\% CI, 4.5-7.3) events per 10,000 person-years, respectively. When broken down according to age and sex, the incidence of all three categories of bleeding event increased with age (Fig. 1b). For hemorrhagic stroke, the incidence was higher in women than in men for individuals aged 50 to 64 years ( 7.5 vs. 1.5 events per 10,000 personyears). However, for patients aged 75 to 84 years, this trend was reversed (5.9 and 9.7 events per 10,000 person-years for women and men, respectively). For UGIB, the incidence was similar in men and women in all age categories, while for LGIB the incidence was higher in women than in men in all age groups (19.1 vs. $16.5,30.2$ vs. 21.8 , and 42.8 vs. 30.0 events per 10,000 person-years for individuals aged 50 to 64 years, 65 to 74 years, and 75 to 84 years, respectively). The overall incidence of bleeding events was 42.4 per

Table 1 Patient demographics and baseline characteristics of the study cohort, and three sets of cases and controls

\begin{tabular}{|c|c|c|c|c|c|c|c|c|}
\hline & & \multirow{2}{*}{$\begin{array}{l}\text { Study cohort } \\
(N=27,707)\end{array}$} & \multicolumn{2}{|c|}{ Hemorrhagic stroke } & \multicolumn{2}{|l|}{ UGIB } & \multicolumn{2}{|l|}{ LGIB } \\
\hline & & & $\begin{array}{l}\text { Cases } \\
(n=70)\end{array}$ & $\begin{array}{l}\text { Controls } \\
(n=1,000)\end{array}$ & $\begin{array}{l}\text { Cases } \\
(n=152)\end{array}$ & $\begin{array}{l}\text { Controls } \\
(n=1,000)\end{array}$ & $\begin{array}{l}\text { Cases } \\
(n=316)\end{array}$ & $\begin{array}{l}\text { Controls } \\
(n=2,000)\end{array}$ \\
\hline \multirow[t]{2}{*}{ Sex } & Male & $18,887(68.2)$ & $45(64.3)$ & $668(66.8)$ & $102(67.1)$ & $681(68.1)$ & $191(60.4)$ & $1,369(68.5)$ \\
\hline & Female & $8,820(31.8)$ & $25(35.7)$ & $332(33.2)$ & $50(32.9)$ & $319(31.9)$ & $125(39.6)$ & $631(31.6)$ \\
\hline \multirow[t]{4}{*}{ Age, years } & $50-59$ & $5,929(21.4)$ & $5(7.1)$ & $59(5.9)$ & $13(8.6)$ & $75(7.5)$ & $30(9.5)$ & $241(12.1)$ \\
\hline & $60-69$ & 9,323 (33.6) & $16(22.9)$ & $237(23.7)$ & $41(27.0)$ & $277(27.7)$ & $82(25.9)$ & $621(31.1)$ \\
\hline & $70-79$ & $9,545(34.4)$ & $30(42.9)$ & $456(45.6)$ & $66(43.4)$ & $436(43.6)$ & $144(45.6)$ & $832(41.6)$ \\
\hline & $80-84$ & $2,910(10.5)$ & $19(27.1)$ & $248(24.8)$ & $32(21.1)$ & $212(21.2)$ & $60(19.0)$ & $306(15.3)$ \\
\hline \multirow[t]{5}{*}{$\mathrm{BMI}, \mathrm{kg} / \mathrm{m}^{2}$} & $<20$ & $632(2.3)$ & $6(8.6)$ & $35(3.5)$ & $6(3.9)$ & $22(2.2)$ & $10(3.2)$ & $61(3.0)$ \\
\hline & $20-24$ & $6,419(23.2)$ & $17(24.3)$ & $249(24.9)$ & $36(23.7)$ & $246(24.6)$ & 65 (20.6) & $441(22.1)$ \\
\hline & $25-29$ & $10,645(38.4)$ & $21(30.0)$ & $413(41.3)$ & $64(42.1)$ & $414(41.4)$ & $139(44.0)$ & $868(43.4)$ \\
\hline & $\geq 30$ & $6,328(22.8)$ & $21(30.0)$ & $258(25.8)$ & $39(25.7)$ & $284(28.4)$ & 89 (28.2) & $546(27.3)$ \\
\hline & Unknown & 3,683 (13.3) & $5(7.1)$ & $45(4.5)$ & $7(4.6)$ & $34(3.4)$ & $13(4.1)$ & $84(4.2)$ \\
\hline \multirow[t]{3}{*}{ History of peptic ulcer disease ${ }^{a}$} & None & $25,077(90.5)$ & $56(80.0)$ & $867(86.7)$ & $109(71.7)$ & $876(87.6)$ & $277(87.7)$ & $1,824(91.2)$ \\
\hline & Uncomplicated & $1,615(5.8)$ & $11(15.7)$ & $96(9.6)$ & $28(18.4)$ & $97(9.7)$ & $27(8.5)$ & $119(6.0)$ \\
\hline & Complicated & $1,015(3.7)$ & $3(4.3)$ & $37(3.7)$ & $15(9.9)$ & $27(2.7)$ & $12(3.8)$ & $57(2.9)$ \\
\hline \multirow[t]{3}{*}{ Qualifying serious coronary event } & Myocardial infarction & $16,107(58.1)$ & $47(67.1)$ & $586(58.6)$ & $81(53.3)$ & $587(58.7)$ & $161(50.9)$ & $1,110(55.5)$ \\
\hline & Unstable angina & $1,919(6.9)$ & $4(5.7)$ & $69(6.9)$ & $14(9.2)$ & $74(7.4)$ & $36(11.4)$ & $140(7.0)$ \\
\hline & Elective revascularization & $9,681(34.9)$ & $19(27.1)$ & $345(34.5)$ & $57(37.5)$ & 339 (33.9) & $119(37.7)$ & $750(37.5)$ \\
\hline
\end{tabular}

Data are presented as $\mathrm{n}(\%)$

$B M I$ body mass index, $L G I B$ lower gastrointestinal bleeding, UGIB upper gastrointestinal bleeding

${ }^{\text {a } D i a g n o s e d ~ a n y ~ t i m e ~ b e f o r e ~ t h e ~ s e r i o u s ~ c o r o n a r y ~ e v e n t ~}$ 

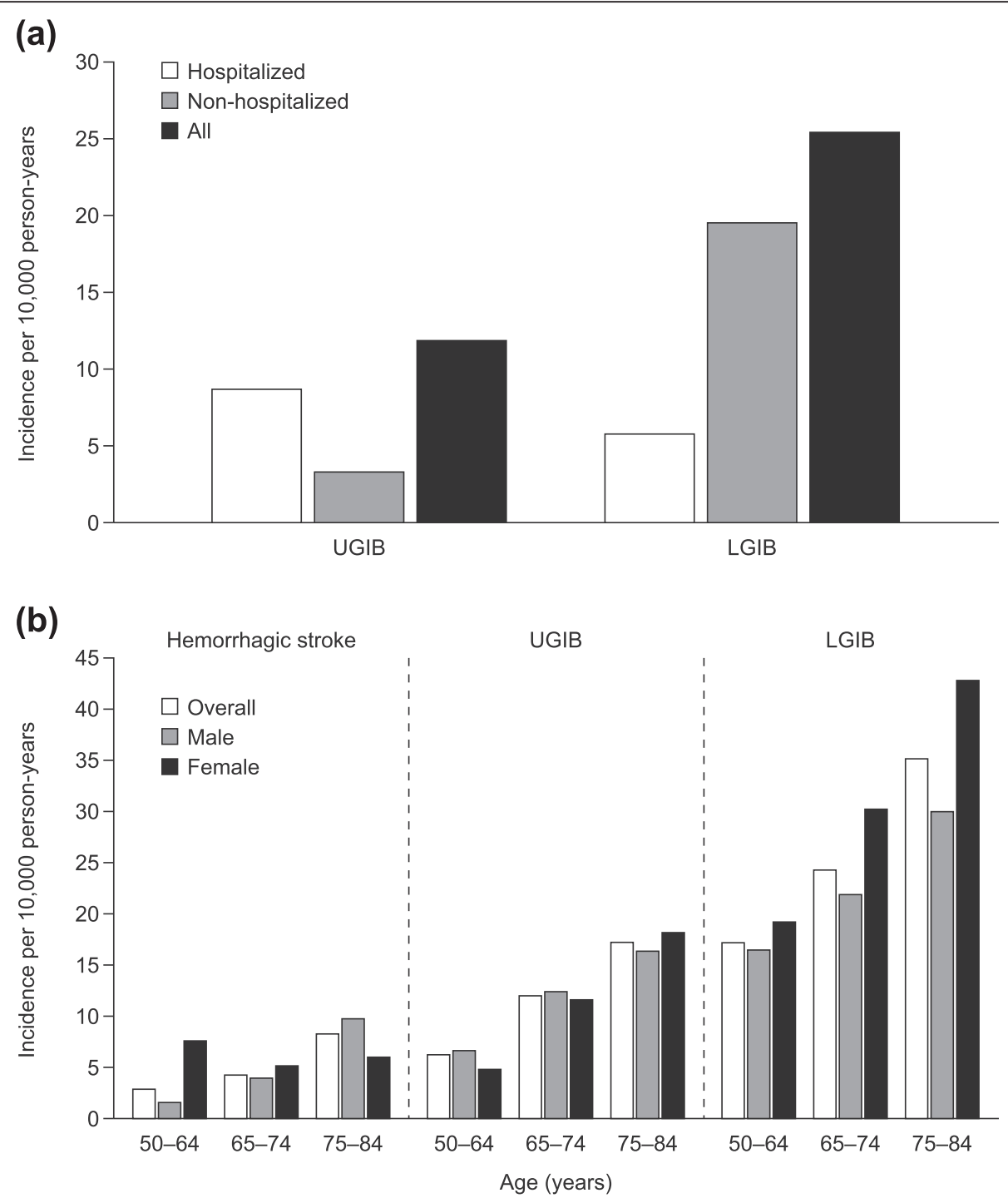

Fig. 1 Incidence of (a) UGIB and LGIB, according to hospitalization status and (b) hemorrhagic stroke, UGIB, and LGIB, according to age and sex. $L G I B$ lower gastrointestinal bleeding, UGIB upper gastrointestinal bleeding

10,000 person-years for any bleeding event and 3.2 cases per 10,000 person-years for fatal bleeding events.

\section{Nested case-control analyses}

In nested case-control analyses, no associations were seen between current use of ASA and risk of hemorrhagic stroke (Table 2) or LGIB (Table 3). A non-significant increased risk of UGIB was observed among current users of ASA (Table 4). For patients receiving a dose of $300 \mathrm{mg}$ $(n=15)$, however, ASA use was associated with a significant increase in the risk of hemorrhagic stroke (OR, 8.23; $95 \%$ CI, 1.77-38.26). No significant associations were seen between the use of clopidogrel and the risk of hemorrhagic stroke. In contrast, current clopidogrel use was associated with a significant increase in the risk of UGIB (OR, 2.17; 95 \% CI, 1.35-3.49) and LGIB (OR, 1.86;
$95 \%$ CI, 1.34-2.57). The use of dual antiplatelet therapy was associated with a significant increase in the risk of UGIB (OR, 2.42; $95 \% \mathrm{CI}, 1.09-5.36$ ) and a nonsignificant increase in the risk of LGIB (OR, 1.63; $95 \%$ CI, 0.95-2.81). Results from nested-control analyses were similar when only hospitalized cases of UGIB and LGIB ( $n=111$ and $n=72$, respectively) were considered (Additional files 3 and 4). Additionally we found that individuals using warfarin (OR, 3.71; $95 \% \mathrm{CI}, 1.66-$ 8.28 ) or combined antithrombotic therapy with warfarin and antiplatelets (OR, 6.36; $95 \%$ CI: 1.34-30.16) experienced a greatly increased risk of hemorrhagic stroke, but not of UGIB or LGIB (Tables 5, 6, and 7).

Other comorbidities and current drug use found to be significantly associated with hemorrhagic stroke, UGIB, and LGIB are listed in Table 8. The largest increase in 
Table 2 Effect of low-dose ASA and clopidogrel on the risk of hemorrhagic stroke

\begin{tabular}{|c|c|c|c|c|}
\hline & $\begin{array}{l}\text { Cases } \\
(n=70) \\
\mathrm{n}(\%)\end{array}$ & $\begin{array}{l}\text { Controls } \\
(n=1,000) \\
\mathrm{n}(\%)\end{array}$ & $\begin{array}{l}\text { Odds ratio }^{a} \\
(95 \% \mathrm{Cl})\end{array}$ & $P$ value \\
\hline \multicolumn{5}{|l|}{ ASA } \\
\hline Non-use & $20(28.6)$ & $164(16.4)$ & $1(-)$ & \\
\hline Current use & $44(62.9)$ & $753(75.3)$ & $0.88(0.42-1.85)$ & 0.74 \\
\hline \multicolumn{5}{|l|}{ Duration } \\
\hline$<1$ month & $3(4.3)$ & $29(2.9)$ & $0.77(0.11-5.56)$ & 0.80 \\
\hline $1-12$ months & $16(22.9)$ & $201(20.1)$ & $0.71(0.26-1.96)$ & 0.51 \\
\hline$\geq 1$ year & $25(35.7)$ & $523(52.3)$ & $1.15(0.50-2.66)$ & 0.74 \\
\hline \multicolumn{5}{|l|}{ Dose } \\
\hline 75 mg/day & $39(55.7)$ & $672(67.2)$ & $0.88(0.41-1.86)$ & 0.74 \\
\hline 150 mg/day & $1(1.4)$ & $70(7.0)$ & $0.19(0.02-1.66)$ & 0.13 \\
\hline 300 mg/day & $4(5.7)$ & $11(1.1)$ & $8.23(1.77-38.26)$ & 0.01 \\
\hline Recent use & $2(2.9)$ & $44(4.4)$ & $0.51(0.10-2.69)$ & 0.43 \\
\hline Past use & $4(5.7)$ & $39(3.9)$ & $1.02(0.30-3.47)$ & 0.98 \\
\hline \multicolumn{5}{|l|}{ Clopidogrel } \\
\hline Non-use & $50(71.4)$ & $757(75.7)$ & $1(-)$ & \\
\hline Current use & $16(22.9)$ & 168 (16.8) & $1.49(0.70-3.17)$ & 0.30 \\
\hline \multicolumn{5}{|l|}{ Duration } \\
\hline$<1$ month & $3(4.3)$ & $18(1.8)$ & $2.09(0.32-13.67)$ & 0.44 \\
\hline $1-12$ months & $7(10.0)$ & $75(7.5)$ & $0.99(0.35-2.78)$ & 0.98 \\
\hline$\geq 1$ year & $6(8.6)$ & $75(7.5)$ & $2.42(0.81-7.22)$ & 0.11 \\
\hline \multicolumn{5}{|l|}{ Dose } \\
\hline 75 mg/day & $16(22.9)$ & 156 (15.6) & $1.61(0.75-3.45)$ & 0.22 \\
\hline$\geq 150$ mg/day & $0(0.0)$ & $12(1.2)$ & & \\
\hline Recent use & $3(4.3)$ & $19(1.9)$ & $1.34(0.33-5.49)$ & 0.68 \\
\hline Past use & $1(1.4)$ & $56(5.6)$ & $0.22(0.03-1.78)$ & 0.16 \\
\hline \multicolumn{5}{|l|}{ Dual antiplatelet therapy } \\
\hline $\begin{array}{l}\text { Non-use of both } \\
\text { ASA and clopidogrel }\end{array}$ & $13(18.6)$ & $96(9.6)$ & $1(-)$ & \\
\hline $\begin{array}{l}\text { Current use of both } \\
\text { ASA and clopidogrel }\end{array}$ & $7(10.0)$ & $91(9.1)$ & $1.20(0.33-4.33)$ & 0.78 \\
\hline $\begin{array}{l}\text { Current ASA use } \\
\text { and non-current } \\
\text { clopidogrel use }\end{array}$ & $37(52.9)$ & $662(66.2)$ & $1.04(0.43-2.55)$ & 0.93 \\
\hline $\begin{array}{l}\text { Current clopidogrel } \\
\text { use and non-current } \\
\text { ASA use }\end{array}$ & $9(12.9)$ & $77(7.7)$ & $2.20(0.72-6.71)$ & 0.17 \\
\hline
\end{tabular}

ASA acetylsalicylic acid, $C l$ confidence interval, NSAID non-steroidal antiinflammatory drug, $P C P$ primary care physician, $P P I$ proton pump inhibitor

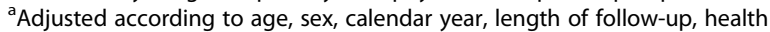
services utilization (PCP visits, referrals and hospitalizations), smoking, type of coronary event, history of peptic ulcer disease, and use of PPIs, ASA, clopidogrel, NSAIDs and warfarin

risk of hemorrhagic stroke was seen in patients who had a history of hemorrhagic stroke before the study period (OR, 23.35; $95 \%$ CI, 5.11-106.73), although this observation was based on a small number of patients. Risk of
Table 3 Effect of low-dose ASA and clopidogrel on the risk of LGIB

\begin{tabular}{|c|c|c|c|c|}
\hline & $\begin{array}{l}\text { Cases } \\
(n=316) \\
\mathrm{n}(\%)\end{array}$ & $\begin{array}{l}\text { Controls } \\
(n=2,000) \\
\mathrm{n}(\%)\end{array}$ & $\begin{array}{l}\text { Odds ratio }^{a} \\
(95 \% \mathrm{Cl})\end{array}$ & $P$ value \\
\hline \multicolumn{5}{|l|}{ ASA } \\
\hline Non-use & $74(23.4)$ & $370(18.5)$ & $1(-)$ & \\
\hline Current use & $222(70.3)$ & $\begin{array}{l}1502 \\
(75.1)\end{array}$ & $0.96(0.68-1.35)$ & 0.80 \\
\hline \multicolumn{5}{|l|}{ Duration } \\
\hline$<1$ month & $11(3.5)$ & $65(3.3)$ & $0.79(0.35-1.80)$ & 0.57 \\
\hline $1-12$ months & $97(30.7)$ & $441(22.1)$ & $1.20(0.79-1.82)$ & 0.40 \\
\hline$\geq 1$ year & $114(36.1)$ & 996 (49.8) & $0.83(0.56-1.22)$ & 0.34 \\
\hline \multicolumn{5}{|l|}{ Dose } \\
\hline 75 mg/day & $193(61.1)$ & $\begin{array}{l}1286 \\
(64.3)\end{array}$ & $0.96(0.68-1.36)$ & 0.81 \\
\hline 150 mg/day & $22(7.0)$ & $178(8.9)$ & $0.90(0.51-1.57)$ & 0.70 \\
\hline 300 mg/day & $7(2.2)$ & $38(1.9)$ & $1.30(0.52-3.21)$ & 0.57 \\
\hline Recent use & $10(3.2)$ & $59(3.0)$ & $1.12(0.51-2.44)$ & 0.77 \\
\hline Past use & $10(3.2)$ & $69(3.5)$ & $0.76(0.36-1.60)$ & 0.47 \\
\hline \multicolumn{5}{|l|}{ Clopidogrel } \\
\hline Non-use & $200(63.3)$ & $\begin{array}{l}1529 \\
(76.5)\end{array}$ & $1(-)$ & \\
\hline Current use & $95(30.1)$ & 318 (15.9) & $1.86(1.34-2.57)$ & $<0.01$ \\
\hline \multicolumn{5}{|l|}{ Duration } \\
\hline$<1$ month & $9(2.8)$ & $25(1.3)$ & $2.40(0.94-6.13)$ & 0.07 \\
\hline 1-12 months & 44 (13.9) & $148(7.4)$ & $1.51(0.97-2.36)$ & 0.07 \\
\hline$\geq 1$ year & $42(13.3)$ & $145(7.3)$ & $2.12(1.37-3.27)$ & $<0.01$ \\
\hline \multicolumn{5}{|l|}{ Dose } \\
\hline 75 mg/day & 84 (26.6) & $286(14.3)$ & $1.82(1.30-2.55)$ & $<0.01$ \\
\hline$\geq 150$ mg/day & $11(3.5)$ & $32(1.6)$ & $2.15(1.01-4.58)$ & 0.05 \\
\hline Recent use & $8(2.5)$ & $40(2.0)$ & $1.30(0.58-2.94)$ & 0.52 \\
\hline Past use & $13(4.1)$ & $113(5.7)$ & $0.80(0.43-1.48)$ & 0.47 \\
\hline \multicolumn{5}{|l|}{ Dual antiplatelet therapy } \\
\hline $\begin{array}{l}\text { Non-use of both } \\
\text { ASA and clopidogrel }\end{array}$ & $40(12.7)$ & $237(11.9)$ & $1(-)$ & \\
\hline $\begin{array}{l}\text { Current use of both } \\
\text { ASA and clopidogrel }\end{array}$ & $57(18.0)$ & $190(9.5)$ & $1.63(0.95-2.81)$ & 0.08 \\
\hline $\begin{array}{l}\text { Current ASA use } \\
\text { and non-current } \\
\text { clopidogrel use }\end{array}$ & $165(52.2)$ & $\begin{array}{l}1312 \\
(65.6)\end{array}$ & $0.84(0.54-1.32)$ & 0.46 \\
\hline $\begin{array}{l}\text { Current clopidogrel } \\
\text { use and non-current } \\
\text { ASA use }\end{array}$ & $38(12.0)$ & $128(6.4)$ & $1.56(0.88-2.74)$ & 0.13 \\
\hline
\end{tabular}

ASA acetylsalicylic acid, $C l$ confidence interval, $L G I B$ lower gastrointestinal bleeding, NSAID non-steroidal anti-inflammatory drug, $P C P$ primary care physician, $P P I$ proton pump inhibitor

aAdjusted according to age, sex, calendar year, length of follow-up, health services utilization (PCP visits, referrals and hospitalizations), smoking, type of coronary event, history of peptic ulcer disease and use of PPIs, ASA, clopidogrel, NSAIDs and warfarin 
Table 4 Effect of low-dose ASA and clopidogrel on the risk of UGIB

\begin{tabular}{|c|c|c|c|c|}
\hline & $\begin{array}{l}\text { Cases } \\
(n=152) \\
n(\%)\end{array}$ & $\begin{array}{l}\text { Controls } \\
(n=1,000) \\
\mathrm{n}(\%)\end{array}$ & $\begin{array}{l}\text { Odds ratio } \\
(95 \% \mathrm{Cl})\end{array}$ & $P$ value \\
\hline \multicolumn{5}{|l|}{ ASA } \\
\hline Non-use & $29(19.1)$ & $186(18.6)$ & $1(-)$ & \\
\hline Current use & $107(70.4)$ & $753(75.3)$ & $1.31(0.76-2.27)$ & 0.33 \\
\hline \multicolumn{5}{|l|}{ Duration } \\
\hline$<1$ month & $8(5.3)$ & $32(3.2)$ & $0.92(0.29-2.91)$ & 0.89 \\
\hline 1-12 months & $38(25.0)$ & $191(19.1)$ & $1.26(0.62-2.57)$ & 0.53 \\
\hline$\geq 1$ year & $61(40.1)$ & $530(53.0)$ & $1.39(0.72-2.67)$ & 0.33 \\
\hline \multicolumn{5}{|l|}{ Dose } \\
\hline 75 mg/day & $98(64.5)$ & $653(65.3)$ & $1.40(0.80-2.43)$ & 0.23 \\
\hline 150 mg/day & $6(3.9)$ & $85(8.5)$ & $0.53(0.18-1.59)$ & 0.26 \\
\hline 300 mg/day & $3(2.0)$ & $15(1.5)$ & $2.47(0.60-10.24)$ & 0.21 \\
\hline Recent use & $10(6.6)$ & $24(2.4)$ & $4.33(1.61-11.66)$ & $<0.01$ \\
\hline Past use & $6(3.9)$ & $37(3.7)$ & $0.85(0.30-2.44)$ & 0.76 \\
\hline \multicolumn{5}{|l|}{ Clopidogrel } \\
\hline Non-use & $96(63.2)$ & $765(76.5)$ & $1(-)$ & \\
\hline Current use & 48 (31.6) & $164(16.4)$ & $2.17(1.35-3.49)$ & $<0.01$ \\
\hline \multicolumn{5}{|l|}{ Duration } \\
\hline$<1$ month & $10(6.6)$ & $11(1.1)$ & $5.76(1.82-18.27)$ & $<0.01$ \\
\hline $1-12$ months & $25(16.4)$ & $73(7.3)$ & $2.14(1.10-4.18)$ & 0.03 \\
\hline$\geq 1$ year & $13(8.6)$ & $80(8.0)$ & $1.80(0.85-3.80)$ & 0.12 \\
\hline \multicolumn{5}{|l|}{ Dose } \\
\hline 75 mg/day & $46(30.3)$ & $154(15.4)$ & $2.22(1.38-3.59)$ & $<0.01$ \\
\hline$\geq 150$ mg/day & $2(1.3)$ & $10(1.0)$ & $1.19(0.22-6.31)$ & 0.84 \\
\hline Recent use & $2(1.3)$ & $13(1.3)$ & $1.12(0.22-5.64)$ & 0.89 \\
\hline Past use & $6(3.9)$ & $58(5.8)$ & $0.64(0.25-1.68)$ & 0.37 \\
\hline \multicolumn{5}{|c|}{ Dual antiplatelet therapy } \\
\hline $\begin{array}{l}\text { Non-use of both } \\
\text { ASA and clopidogrel }\end{array}$ & 18 (11.8) & 119 (11.9) & $1(-)$ & \\
\hline $\begin{array}{l}\text { Current use of } \\
\text { both ASA and } \\
\text { clopidogrel }\end{array}$ & $36(23.7)$ & $100(10.0)$ & $2.42(1.09-5.36)$ & 0.03 \\
\hline $\begin{array}{l}\text { Current ASA use } \\
\text { and non-current } \\
\text { clopidogrel use }\end{array}$ & $71(46.7)$ & $653(65.3)$ & $0.91(0.46-1.80)$ & 0.78 \\
\hline $\begin{array}{l}\text { Current clopidogrel } \\
\text { use and non- } \\
\text { current ASA use }\end{array}$ & $12(7.9)$ & $64(6.4)$ & $1.29(0.52-3.20)$ & 0.58 \\
\hline
\end{tabular}

ASA acetylsalicylic acid, $C l$ confidence interval, NSAID non-steroidal antiinflammatory drug, $P C P$ primary care physician, $P P I$ proton pump inhibitor, UGIB upper gastrointestinal bleeding

${ }^{a}$ Adjusted according to age, sex, calendar year, length of follow-up, health services utilization (PCP visits, referrals and hospitalizations), smoking, type of coronary event, history of peptic ulcer disease and use of PPIs, ASA, clopidogrel, NSAIDs and warfarin

hemorrhagic stroke was also increased in patients who had been hospitalized at least once for any reason in the year before the index date, and in those who were
Table 5 Effect of warfarin and antithrombotics on the risk of hemorrhagic stroke

\begin{tabular}{|c|c|c|c|c|}
\hline & $\begin{array}{l}\text { Cases } \\
(n=70) \\
\mathrm{n}(\%)\end{array}$ & $\begin{array}{l}\text { Controls } \\
(n=1000) \\
\mathrm{n}(\%)\end{array}$ & $\begin{array}{l}\text { Odds ratios } \\
(95 \% \mathrm{Cl})\end{array}$ & $P$ value \\
\hline \multicolumn{5}{|l|}{ Warfarin } \\
\hline Non-use ${ }^{b}$ & $50(71.4)$ & $904(90.4)$ & $1(-)$ & \\
\hline Current use & $17(24.3)$ & $83(8.3)$ & $3.71(1.66-8.28)$ & $<0.01$ \\
\hline$<1$ month & $1(1.4)$ & $4(0.4)$ & $2.99(0.27-32.88)$ & 0.37 \\
\hline 1-12 months & $5(7.1)$ & $30(3.0)$ & $2.14(0.64-7.08)$ & 0.21 \\
\hline$>1$ year & $11(15.7)$ & $49(4.9)$ & $6.26(2.37-16.52)$ & $<0.01$ \\
\hline Recent use & $2(2.9)$ & $5(0.5)$ & $9.21(1.43-59.26)$ & 0.02 \\
\hline Past use & $1(1.4)$ & $8(0.8)$ & $3.26(0.34-31.38)$ & 0.31 \\
\hline \multicolumn{5}{|l|}{ Antithrombotic } \\
\hline $\begin{array}{l}\text { Non-use AP and } \\
\text { non-use warfarin }\end{array}$ & $3(4.3)$ & $44(4.4)$ & $1(-)$ & \\
\hline $\begin{array}{l}\text { Current AP and } \\
\text { non-current warfarin }\end{array}$ & $46(65.7)$ & $810(81.0)$ & $0.93(0.26-3.32)$ & 0.92 \\
\hline $\begin{array}{l}\text { Non-current AP and } \\
\text { current warfarin }\end{array}$ & $10(14.3)$ & $63(6.3)$ & $2.26(0.54-9.47)$ & 0.26 \\
\hline $\begin{array}{l}\text { Current AP and } \\
\text { current warfarin }\end{array}$ & $7(10.0)$ & $20(2.0)$ & $6.36(1.34-30.16)$ & 0.02 \\
\hline
\end{tabular}

\section{$A P$ antiplatelets}

aEstimates adjusted by age, sex, calendar year, time of follow up after serious coronary event, health services utilisation, smoking, proton pump inhibitor, antithrombotic and nonsteroidal anti-inflammatory drug use, type of serious coronary event and prior peptic ulcer disease using a logistic regression model ${ }^{\mathrm{b}}$ Reference category

currently using any NSAID. UGIB was most strongly associated with having valvular heart disease (OR, 3.73; $95 \% \mathrm{CI}, 1.95-7.11)$ or complicated peptic ulcer disease (OR, 3.71; $95 \% \mathrm{CI}, 1.76-7.84)$. The strongest association with risk of LGIB was seen for pancreatic disease (OR, 4.49; 95 \% CI, 1.20-16.74), although this observation was based on only a small number of patients.

\section{Discussion}

We have shown that, in the period 2000 to 2007, among nearly 30,000 patients who were hospitalized for a serious coronary event (myocardial infarction, revascularization of the coronary arteries, or unstable angina) and who were alive 30 days later, the incidence of hemorrhagic stroke, UGIB, and LGIB up to 2011 was 5.0, 11.9, and 25.5 events per 10,000 person-years, respectively. Incidence increased with age for all three categories of bleeding events. To our knowledge, this is the first population-based study to report absolute risk of these three types of bleeding events in patients hospitalized for a coronary event. The use of a primary care database affords valuable insight into the burden of bleeding events and our results suggest that the sole use of hospital records for identification of gastrointestinal bleeding events may severely underestimate their incidence, particularly for LGIB. In our study, more than three quarters of 
Table 6 Effect of warfarin and antithrombotics on the risk of lower gastrointestinal bleeding

\begin{tabular}{|c|c|c|c|c|}
\hline & $\begin{array}{l}\text { Cases } \\
(n=316) \\
n(\%)\end{array}$ & $\begin{array}{l}\text { Controls } \\
(n=2000) \\
n(\%)\end{array}$ & $\begin{array}{l}\text { Odds ratios }{ }^{a} \\
(95 \% \mathrm{Cl})\end{array}$ & $P$ value \\
\hline \multicolumn{5}{|l|}{ Warfarin } \\
\hline Non-use ${ }^{b}$ & $276(87.3)$ & 1813 (90.6) & $1(-)$ & \\
\hline Current use & $32(10.1)$ & $157(7.8)$ & $1.10(0.69-1.78)$ & 0.68 \\
\hline$<1$ month & $3(0.9)$ & $8(0.4)$ & $1.55(0.38-6.34)$ & 0.54 \\
\hline 1-12 months & $15(4.7)$ & $44(2.2)$ & $1.78(0.89-3.55)$ & 0.10 \\
\hline$>1$ year & $14(4.4)$ & $105(5.3)$ & $0.73(0.38-1.40)$ & 0.34 \\
\hline Recent use & $2(0.6)$ & $11(0.5)$ & $0.91(0.19-4.42)$ & 0.91 \\
\hline Past use & $6(1.9)$ & $19(0.9)$ & $1.31(0.49-3.46)$ & 0.59 \\
\hline \multicolumn{5}{|l|}{ Antithrombotic } \\
\hline $\begin{array}{l}\text { Non-use AP and } \\
\text { non-use warfarin }\end{array}$ & $18(5.7)$ & $128(6.4)$ & $1(-)$ & \\
\hline $\begin{array}{l}\text { Current AP and } \\
\text { non-current warfarin }\end{array}$ & $252(79.7)$ & 1596 (79.8) & $1.00(0.59-1.71)$ & 1.00 \\
\hline $\begin{array}{l}\text { Non-current AP and } \\
\text { current warfarin }\end{array}$ & $24(7.6)$ & $116(5.8)$ & $1.01(0.50-2.02)$ & 0.99 \\
\hline $\begin{array}{l}\text { Current AP and } \\
\text { current warfarin }\end{array}$ & $8(2.5)$ & $41(2.1)$ & $0.89(0.35-2.30)$ & 0.82 \\
\hline
\end{tabular}

$A P$ antiplatelets

${ }^{a}$ Estimates adjusted by age, sex, calendar year, time of follow up after serious coronary event, health services utilisation, smoking, proton pump inhibitor, antithrombotic and nonsteroidal anti-inflammatory drug use, type of serious coronary event and prior peptic ulcer disease using a logistic regression model ${ }^{\mathrm{b}}$ Reference category

patients with LGIB were referred to a consultant, but not hospitalized.

With regard to drug use, we did not observe statistically significant associations between the risk of hemorrhagic stroke and current use of low-dose ASA for doses of $75 \mathrm{mg}$ or $150 \mathrm{mg}$ per day, relative to nonuse. A dose of $300 \mathrm{mg}$ per day, however, was associated with a significant increase in the risk of hemorrhagic stroke, but this association was based on a small number of users. Current use of clopidogrel, with or without concomitant low-dose ASA, was not significantly associated with the risk of hemorrhagic stroke. However, the combined use of warfarin and antiplatelets was associated with a greatly increased risk of hemorrhagic stroke.

Current use of clopidogrel was associated with a significantly increased risk of UGIB and LGIB. Current use of low-dose ASA did not show a significant association with either UGIB or LGIB. Dual antiplatelet therapy was associated with a significant increase in the risk of UGIB and a non-significant increase in the risk of LGIB.

The increased risk of UGIB and LGIB seen among patients receiving clopidogrel, and the significant association between UGIB and the use of dual antiplatelet therapy, are in line with the previous evidence that use of antiplatelet therapies increases the risk of bleeding events $[6-8,10-12]$. Previous studies have reported an
Table 7 Effect of warfarin and antithrombotics on the risk of upper gastrointestinal bleeding

\begin{tabular}{|c|c|c|c|c|}
\hline & $\begin{array}{l}\text { Cases } \\
(n=152) \\
\mathrm{n}(\%)\end{array}$ & $\begin{array}{l}\text { Controls } \\
(n=1000) \\
n(\%)\end{array}$ & $\begin{array}{l}\text { Odds ratios } \\
(95 \% \mathrm{Cl})\end{array}$ & $P$ value \\
\hline \multicolumn{5}{|l|}{ Warfarin } \\
\hline Non-use ${ }^{b}$ & $128(84.2)$ & $887(88.7)$ & $1(-)$ & \\
\hline Current use & $23(15.1)$ & $102(10.2)$ & $1.79(0.94-3.41)$ & 0.08 \\
\hline$<1$ month & $5(3.3)$ & $5(0.5)$ & $7.76(1.69-35.68)$ & 0.01 \\
\hline 1-12 months & $8(5.3)$ & $43(4.3)$ & $0.99(0.40-2.47)$ & 0.98 \\
\hline$>1$ year & $10(6.6)$ & $54(5.4)$ & $2.11(0.83-5.33)$ & 0.11 \\
\hline Recent use & $1(0.7)$ & $5(0.5)$ & $1.83(0.19-17.60)$ & 0.60 \\
\hline Past use & $0(0.0)$ & $6(0.6)$ & - & \\
\hline \multicolumn{5}{|l|}{ Antithrombotic } \\
\hline $\begin{array}{l}\text { Non-use AP and } \\
\text { non-use warfarin }\end{array}$ & $10(6.6)$ & $55(5.5)$ & $1(-)$ & \\
\hline $\begin{array}{l}\text { Current AP and } \\
\text { non-current warfarin }\end{array}$ & $107(70.4)$ & $789(78.9)$ & $0.68(0.31-1.49)$ & 0.34 \\
\hline $\begin{array}{l}\text { Non-current AP and } \\
\text { current warfarin }\end{array}$ & $11(7.2)$ & $74(7.4)$ & $0.66(0.24-1.85)$ & 0.43 \\
\hline $\begin{array}{l}\text { Current AP and } \\
\text { current warfarin }\end{array}$ & $12(7.9)$ & $28(2.8)$ & $1.67(0.56-4.96)$ & 0.35 \\
\hline
\end{tabular}

AP antiplatelets

a Estimates adjusted by age, sex, calendar year, time of follow up after serious coronary event, health services utilisation, smoking, proton pump inhibitor, antithrombotic and nonsteroidal anti-inflammatory drug use, type of serious coronary event and prior peptic ulcer disease using a logistic regression model ${ }^{\mathrm{b}}$ Reference category

increased risk of bleeding events with clopidogrel combined with low-dose ASA therapy compared with monotherapy. In a randomized study in patients with non-ST-segment elevation myocardial infarctions, major bleeding events were significantly more common with clopidogrel combined with ASA than with ASA alone (relative risk [RR], 1.38; 95 \% CI, 1.13-1.67). Major gastrointestinal bleeding events were more frequent with dual antiplatelet therapy than with ASA monotherapy, but frequency of intracranial bleeding events did not differ between the groups [6]. In another randomized study, dual antiplatelet therapy was associated with significantly higher rates of major gastrointestinal bleeding and of intracranial bleeding than clopidogrel monotherapy in high-risk patients following a recent ischemic stroke or transient ischemic attack [25].

A previous analysis of individuals with UGIB identified from THIN showed that dual antiplatelet therapy with clopidogrel in combination with low-dose ASA was associated with an increased risk of UGIB compared with low-dose ASA alone (RR, 2.08; 95 \% CI, 1.34-3.21). Current clopidogrel use was also associated with an increased risk of UGIB compared with non-use, as was the current use of low-dose ASA [11]. An analysis using the National Health Insurance Database in Taiwan identified clopidogrel use as a significant factor for increased risk 
Table 8 Comorbidities and current drug use significantly associated with hemorrhagic stroke, UGIB and LGIB

\begin{tabular}{|c|c|c|c|c|}
\hline & $\begin{array}{l}\text { Cases } \\
\mathrm{n}(\%) \\
\end{array}$ & $\begin{array}{l}\text { Controls } \\
\mathrm{n}(\%)\end{array}$ & $\begin{array}{l}\text { Odds ratio } \\
(95 \% \mathrm{Cl}) \\
\end{array}$ & $P$ value \\
\hline \multicolumn{5}{|l|}{ Hemorrhagic stroke $(n=70)$} \\
\hline \multicolumn{5}{|l|}{ Comorbidities $^{\mathrm{b}}$} \\
\hline History of hemorrhagic stroke & $4(5.7)$ & $5(0.5)$ & $23.35(5.11-106.73)$ & $<0.01$ \\
\hline$\geq 1$ hospitalization in the year before index date ${ }^{c}$ & $35(50.0)$ & $282(28.2)$ & $1.93(1.06-3.49)$ & 0.03 \\
\hline \multicolumn{5}{|l|}{ Current drug use $^{d}$} \\
\hline Diuretics & $21(30.0)$ & $376(37.6)$ & $0.52(0.28-0.99)$ & 0.05 \\
\hline NSAIDs & $9(12.9)$ & $70(7.0)$ & $2.53(1.12-5.68)$ & 0.03 \\
\hline \multicolumn{5}{|l|}{ UGIB $(n=152)$} \\
\hline \multicolumn{5}{|l|}{ Comorbidities $^{\mathrm{b}}$} \\
\hline Complicated peptic ulcer disease & $15(9.9)$ & $27(2.7)$ & $3.71(1.76-7.84)$ & $<0.01$ \\
\hline Uncomplicated peptic ulcer disease & $28(18.4)$ & $97(9.7)$ & $1.71(1.02-2.86)$ & 0.04 \\
\hline Valvular heart disease & $22(14.5)$ & $50(5.0)$ & $3.73(1.95-7.11)$ & $<0.01$ \\
\hline$\geq 1$ hospitalization in year before index date ${ }^{c}$ & $84(55.3)$ & $262(26.2)$ & $2.50(1.62-3.86)$ & $<0.01$ \\
\hline \multicolumn{5}{|l|}{ Current drug use $^{d}$} \\
\hline NSAIDs & $23(15.1)$ & $80(8.0)$ & $2.25(1.27-3.96)$ & 0.01 \\
\hline PPIs & $68(44.7)$ & $316(31.6)$ & $1.62(1.06-2.47)$ & 0.03 \\
\hline \multicolumn{5}{|l|}{$\operatorname{LGIB}(n=316)$} \\
\hline \multicolumn{5}{|l|}{ Comorbidities $^{a}$} \\
\hline Pancreatic disease & $4(1.3)$ & $8(0.4)$ & $4.49(1.20-16.74)$ & 0.03 \\
\hline Unstable angina & $75(23.7)$ & $283(14.1)$ & $1.86(1.25-2.77)$ & $<0.01$ \\
\hline Dyspepsia & $104(32.9)$ & $390(19.5)$ & $1.64(1.23-2.18)$ & $<0.01$ \\
\hline Diabetes mellitus & $46(14.6)$ & $343(17.2)$ & $0.62(0.43-0.88)$ & 0.01 \\
\hline$\geq 1$ hospitalization in year before index date ${ }^{c}$ & $142(44.9)$ & $575(28.7)$ & $1.33(0.98-1.79)$ & 0.06 \\
\hline \multicolumn{5}{|l|}{ Current drug use ${ }^{d}$} \\
\hline Calcium-channel blockers & $112(35.4)$ & $544(27.2)$ & $1.34(1.02-1.75)$ & 0.03 \\
\hline NSAIDs & $39(12.3)$ & $167(8.3)$ & $1.52(1.02-2.25)$ & 0.04 \\
\hline PPIs & $147(46.5)$ & $621(31.1)$ & $1.60(1.22-2.10)$ & $<0.01$ \\
\hline \multicolumn{5}{|c|}{$\begin{array}{l}\text { ASA acetylsalicylic acid, CI confidence interval, DVT deep vein thrombosis, LGIB lower gastrointestinal bleeding, NSAID non-steroidal anti-inflammatory drug, PCP } \\
\text { primary care physician, PPI proton pump inhibitor, UGIB upper gastrointestinal bleeding } \\
\text { aAdjusted according to age, sex, calendar year, length of follow-up, health services utilization (PCP visits, referrals and hospitalizations), smoking, type of coronary } \\
\text { event, history of peptic ulcer disease and use of PPIs, ASA, clopidogrel, NSAIDs and warfarin } \\
\text { belative to absence of the respective comorbidity } \\
\text { 'Relative to none } \\
\text { dRelative to non-use }\end{array}$} \\
\hline
\end{tabular}

of both UGIB (hazard ratio [HR], 3.66; $95 \% \mathrm{CI}, 2.96-$ 4.51) and LGIB (HR, 3.52; $95 \%$ CI, 2.74-4.52) [26]. Similarly, a meta-analysis of randomized controlled trials showed that low-dose ASA was associated with a significant increase in the risk of major gastrointestinal bleeding compared with placebo [27]. An increased risk of UGIB was also reported with use of low-dose ASA in meta-analyses of randomized controlled trials and of observational studies [28].

This failure to detect a statistically significant increased risk of UGIB or LGIB associated with ASA in our study could be related to the nature of the study cohort. We started following up patients who had just been hospitalized for a serious coronary event. ASA is generally started in these patients as a result of this event, and most individuals continue to receive ASA therapy for years. In fact, current ASA use was over $75 \%$ among controls and only about $10 \%$ never used ASA between their start and index dates. The relatively small proportion ( $25 \%$ ) of those who were not current users of ASA were mostly discontinuers and are likely to differ in many ways from the rest; their background risk of gastrointestinal bleeding could be much higher than non-users of ASA in the general population. This could explain the absence of an increased risk of UGIB or LGIB with low-dose ASA in our population. 
Our study showed a significant association between the risk of hemorrhagic stroke and the use of ASA $300 \mathrm{mg}$ per day, consistent with findings from previous studies. A meta-analysis of 11 randomized controlled trials reported a significantly increased risk of intracranial bleeding with low-dose ASA compared with placebo (RR, 1.65; 95 \% CI, 1.12-2.44) [27]. However, this analysis showed no evidence for differences in the risk of intracranial bleeding (or other bleeding endpoints) between lower-dose (75-162.5 mg per day) and higher-dose (>162.5-325 mg per day) ASA, although it was limited by the small number of patients receiving higher-dose ASA. In contrast, results from a previous population-based study involving 3,137 cases of hemorrhagic stroke showed that long-term use of low-dose ASA was associated with a decreased risk of subarachnoid hemorrhage [29]. The small number of events in the current study did not allow for separate analyses by type of hemorrhagic stroke.

We have shown that individuals using combined antithrombotic therapy with warfarin and antiplatelets had a greatly increased risk of hemorrhagic stroke. In our previous population-based study we observed a similar six-fold increased risk of intracerebral hemorrhage among individuals using warfarin along with two or more antiplatelet drugs [29]. These new data seem to confirm that the increased risk of hemorrhagic stroke observed for combined antithrombotic therapy exceeds the risk of warfarin alone. Furthermore, a recent metaanalysis of randomized trials and observational studies in patients undergoing percutaneous coronary intervention found that the incidence of major bleeding was higher among individuals using triple therapy (combined use of two antiplatelets with an oral anticoagulant) than among those using dual antiplatelet therapy [30].

Although our nested case-control analyses were adjusted for known risk factors for bleeding events, we cannot rule out some residual confounding by indication. It remains possible that patients at increased risk of hemorrhagic stroke, UGIB, or LGIB are less likely to be prescribed low-dose ASA owing to the known risk associated with its use. Confounding by indication is also a possible explanation for the increased risk of UGIB and LGIB observed for current users of PPIs in our study. PPIs are recommended for co-prescription with antiplatelet therapies among patients at high risk of gastrointestinal bleeding to reduce this risk [10]. Patients may be prescribed a PPI because they have a higher underlying risk of gastrointestinal bleeding, and may therefore have a higher incidence of bleeding than those not prescribed a PPI.

Current use of NSAIDs was associated with an increased risk of UGIB and LGIB. This is in line with results from previous studies that showed an increased risk of gastrointestinal complications associated with the use of NSAIDs
$[10,31]$. A recent meta-analysis of randomized studies also showed a significantly increased risk of upper gastrointestinal complications with all NSAID regimens [32].

The present study has the strength of a large study population selected from a primary care database that is representative of the UK population and has been validated for use in epidemiological studies [18, 21]. It should be noted that THIN does not report use of overthe-counter (OTC) medications. However, prescription medications are free for patients aged 60 years or older in the UK and health care is easily accessed, which is likely to encourage prescription rather than OTC medication use. In our study, most patients (78.6\%) were aged 60 years or older and therefore misclassification of drug use due to medications being obtained OTC should not greatly affect our results. We did not include bleeding events that occurred while hospitalized, which might underestimate bleeding rates in this population. In common with all observational studies, another limitation is the possibility of confounding factors. Although the analyses were adjusted for a number of factors in order to control for this as much as possible, it is not possible to control for all potential confounding factors, so there is a possibility that this may have affected the study findings.

\section{Conclusions}

The results of our study showed that for patients previously hospitalized for a serious coronary event and treated with antiplatelet drugs, the incidence of hemorrhagic stroke and gastrointestinal bleed ranged from 5 to 25 events per 10,000 person-years. Current use of low-dose ASA was not associated with an increased risk of hemorrhagic stroke or LGIB, but was associated with a non-significant increased risk of UGIB. In our study, however, non-users of ASA were a small subgroup of the cohort and were for the most part ASA discontinuers and are likely to have a much higher background risk of gastrointestinal bleeding than the general population; this may explain the failure to observe a significantly increased risk of UGIB and LGIB associated with the use of ASA. Additionally we showed that combined antithrombotic therapy with warfarin and antiplatelet drugs greatly increases the risk of hemorrhagic stroke in this population.

\section{Additional files}

Additional file 1: Information about the sites of UGIB, overall and stratified by hospitalization status. (DOCX $29 \mathrm{~kb}$ )

Additional file 2: Information about the causes of $L G I B$, overall and stratified by hospitalization status. (DOCX $29 \mathrm{~kb}$ )

Additional file 3: information about the effect of low-dose ASA and clopidogrel on the risk of UGIB in hospitalized cases only. (DOCX $30 \mathrm{~kb}$ ) 
Additional file 4: Information about the effect of low-dose ASA and clopidogrel on the risk of LGIB in hospitalized cases only. (DOCX $30 \mathrm{~kb}$ )

Additional file 5: Information about the effects of comorbidities and the risk of hemorrhagic stroke. (DOCX $40 \mathrm{~kb}$ )

Additional file 6: Information about the effects of other drug use and the risk of hemorrhagic stroke. (DOCX $41 \mathrm{~kb}$ )

Additional file 7: Information about the effects of comorbidity and risk of LGIB. (DOCX $41 \mathrm{~kb}$ )

Additional file 8: Information about the effects of other drug use and the risk of LGIB. (DOCX $43 \mathrm{~kb}$ )

Additional file 9: Information about the effects of comorbidities and the risk of UGIB. (DOCX $39 \mathrm{~kb})$

Additional file 10: Information about the effects of other drug use and the risk of UGIB. (DOCX $43 \mathrm{~kb}$ )

\section{Acknowledgements}

The authors would like to thank Prof Angel Lanas, University of Zaragoza, Zaragoza, Spain, for his assistance in this project and particularly for his help in ascertaining gastrointestinal bleeding cases.

\section{Funding}

This study was funded with financial research support from AstraZeneca R\&D, Mölndal, Sweden. Medical writing support was provided by Stéphane Pintat, PhD, of Oxford PharmaGenesis, Oxford, UK and was funded by AstraZeneca R\&D, Mölndal, Sweden.

\section{Availability of data and materials}

Data supporting our findings can be found in the main manuscript and tables, as well as in the Additional files 1, 2, 3, 4, 5, 6, 7, 8, 9 and 10 .

\section{Authors' contributions}

AGP designed the study, performed the statistical analysis, was involved in the interpretation of the data, revised the intellectual content of the manuscript, and approved the final version. MES designed the study, performed the statistical analysis, was involved in the interpretation of the data, revised the intellectual content of the manuscript, and approved the final version. SJ provided input on the design of the study, was involved in the analysis and interpretation of the data, revised the intellectual content of the manuscript, and approved the final version. AH provided input on the design of the study, was involved in the analysis and interpretation of the data, revised the intellectual content of the manuscript, and approved the final version. LAGR designed the study, performed the statistical analysis, was involved in the interpretation of the data, revised the intellectual content of the manuscript, and approved the final version. The manuscript was reviewed by AstraZeneca before submission.

\section{Competing interests}

AGP, MES, and LAGR work for the Spanish Centre for Pharmacoepidemiologic Research (CEIFE), which has received research funding from AstraZeneca R\&D, Mölndal, Sweden and Bayer Pharma AG, Berlin, Germany. LAGR has also received honoraria for serving on scientific advisory boards for Bayer. SJ and $\mathrm{AH}$ are employees of AstraZeneca R\&D, Mölndal, Sweden.

\section{Consent for publication}

Not applicable (the manuscript does not contain any individual person's data).

\section{Ethics approval and consent to participate}

The company that owns THIN (Cegedim Strategic Data) has received ethical approval from the South East Research Ethics Committee to supply anonymized, pre-collected, primary care data for scientific research. Patients can opt out of having their depersonalized records collected; therefore, patient consent is not required when working with anonymized records in THIN. This study was approved by a multicenter research ethics committee (REC reference: 09/H0305/90).

\section{Author details}

${ }^{1}$ Spanish Centre for Pharmacoepidemiologic Research (CEIFE), Madrid, Spain.

${ }^{2}$ Andalusian Bioinformatics Research Centre (CAEBi), Seville, Spain.

${ }^{3}$ AstraZeneca R\&D, Mölndal, Sweden.

Received: 27 August 2015 Accepted: 19 August 2016

Published online: 30 August 2016

\section{References}

1. Allender $S$, Scarborough $P$, Peto $V$, Rayner $M$, Leal L, Luengo-Fernandez $R$, et al. European cardiovascular disease statistics. http://www.ehnheart.org/cvdstatistics.html. Accessed 10 Feb 2015.

2. Hamm CW, Bassand JP, Agewall S, Bax J, Boersma E, Bueno H, et al. ESC guidelines for the management of acute coronary syndromes in patients presenting without persistent ST-segment elevation: the task force for the management of acute coronary syndromes (ACS) in patients presenting without persistent ST-segment elevation of the European society of cardiology (ESC). Eur Heart J. 2011;32:2999-3054.

3. Steg PG, James SK, Atar D, Badano LP, Blömstrom-Lundqvist C, Borger MA, et al. ESC guidelines for the management of acute myocardial infarction in patients presenting with ST-segment elevation: the task force on the management of ST-segment elevation acute myocardial infarction of the European society of cardiology (ESC). Eur Heart J. 2012;33:2569-619.

4. Windecker S, Kolh P, Alfonso F, Collet JP, Cremer J, Falk V, et al. 2014 ESC/ EACTS guidelines on myocardial revascularization: the task force on myocardial revascularization of the European society of cardiology (ESC) and the European association for cardio-thoracic surgery (EACTS). developed with the special contribution of the European association of percutaneous cardiovascular interventions (EAPCI). Eur Heart J. 2014:35:2541-619.

5. Baigent C, Blackwell L, Collins R, Emberson J, Godwin J, Peto R, et al. Aspirin in the primary and secondary prevention of vascular disease: collaborative meta-analysis of individual participant data from randomised trials. Lancet. 2009;373:1849-60.

6. Yusuf S, Zhao F, Mehta SR, Chrolavicius S, Tognoni G, Fox KK. Effects of clopidogrel in addition to aspirin in patients with acute coronary syndromes without ST-segment elevation. N Engl J Med. 2001:345:494-502.

7. Wiviott SD, Braunwald E, McCabe CH, Montalescot G, Ruzyllo W, Gottlieb S, et al. Prasugrel versus clopidogrel in patients with acute coronary syndromes. N Engl J Med. 2007;357:2001-15.

8. Wallentin L, Becker RC, Budaj A, Cannon CP, Emanuelsson H, Held C, et al. Ticagrelor versus clopidogrel in patients with acute coronary syndromes. $\mathrm{N}$ Engl J Med. 2009;361:1045-57.

9. Chen ZM, Jiang LX, Chen YP, Xie JX, Pan HC, Peto R, et al. Addition of clopidogrel to aspirin in 45852 patients with acute myocardial infarction: randomised placebo-controlled trial. Lancet. 2005;366:1607-21.

10. Bhatt DL, Scheiman J, Abraham NS, Antman EM, Chan FK, Furberg CD, et al. ACCF/ACG/AHA 2008 expert consensus document on reducing the gastrointestinal risks of antiplatelet therapy and NSAID use: a report of the American college of cardiology foundation task force on clinical expert consensus documents. Circulation. 2008;118:1894-909.

11. García Rodríguez LA, Lin KJ, Hernández-Díaz S, Johansson S. Risk of upper gastrointestinal bleeding with low-dose acetylsalicylic acid alone and in combination with clopidogrel and other medications. Circulation. 2011;123: 1108-15.

12. Weil J, Colin-Jones D, Langman M, Lawson D, Logan R, Murphy M, et al. Prophylactic aspirin and risk of peptic ulcer bleeding. BMJ. 1995:310:827-30

13. Casado Arroyo R, Polo-Tomas M, Roncales MP, Scheiman J, Lanas A. Lower Gl bleeding is more common than upper among patients on dual antiplatelet therapy: long-term follow-up of a cohort of patients commonly using PPI co-therapy. Heart. 2012;98:718-23.

14. Hashash JG, Shamseddeen W, Skoury A, Aoun N, Barada K. Gross lower gastrointestinal bleeding in patients on anticoagulant and/or antiplatelet therapy: endoscopic findings, management, and clinical outcomes. J Clin Gastroenterol. 2009;43:36-42

15. Nagata N, Niikura R, Aoki T, Shimbo T, Kishida Y, Sekine K, et al. Lower Gl bleeding risk of nonsteroidal anti-inflammatory drugs and antiplatelet drug use alone and the effect of combined therapy. Gastrointest Endosc. 2014:80:1124-31.

16. Abraham NS, Hartman C, Richardson P, Castillo D, Street Jr RL, Naik AD. Risk of lower and upper gastrointestinal bleeding, transfusions, and 
hospitalizations with complex antithrombotic therapy in elderly patients. Circulation. 2013;128:1869-77.

17. Cegedim Strategic Data Medical Research UK. THIN electronic medical record data resource. http://csdmruk.cegedim.com/. Accessed 10 Feb 2015.

18. Bourke A, Dattani H, Robinson M. Feasibility study and methodology to create a quality-evaluated database of primary care data. Inform Prim Care. 2004;12:171-7

19. O'Neil M, Payne C, Read J. Read Codes Version 3: a user led terminology. Methods Inf Med. 1995;34:187-92.

20. In Practice Systems Ltd - Gemscript.http://www.inps4.co.uk/my-vision/ news/gemscript-\%C3\%82\%C2\%96-new-dmd-drug-dictionary-vision. Accessed 10 Feb 2015

21. Lewis JD, Schinnar R, Bilker WB, Wang X, Strom BL. Validation studies of the health improvement network (THIN) database for pharmacoepidemiology research. Pharmacoepidemiol Drug Saf. 2007:16:393-401.

22. García Rodríguez LA, Johansson S, Cea SL. Use of clopidogrel and proton pump inhibitors after a serious acute coronary event: risk of coronary events and peptic ulcer bleeding. Thromb Haemost. 2013;110:1014-24.

23. Gaist D, Wallander MA, González-Pérez A, García Rodríguez LA. Incidence of hemorrhagic stroke in the general population: validation of data from the health improvement network. Pharmacoepidemiol Drug Saf. 2013;22:176-82.

24. García Rodríguez LA, Barreales TL. Risk of upper gastrointestinal complications among users of traditional NSAIDs and COXIBs in the general population. Gastroenterology. 2007;132:498-506.

25. Diener HC, Bogousslavsky J, Brass LM, Cimminiello C, Csiba L, Kaste M, et al. Aspirin and clopidogrel compared with clopidogrel alone after recent ischaemic stroke or transient ischaemic attack in high-risk patients (MATCH): randomised, double-blind, placebo-controlled trial. Lancet. 2004;364:331-7.

26. Lin CC, Hu HY, Luo JC, Peng YL, Hou MC, Lin HC, et al. Risk factors of gastrointestinal bleeding in clopidogrel users: a nationwide populationbased study. Aliment Pharmacol Ther. 2013;38:1119-28.

27. McQuaid KR, Laine L. Systematic review and meta-analysis of adverse events of low-dose aspirin and clopidogrel in randomized controlled trials. Am J Med. 2006;1 19:624-38.

28. Valkhoff VE, Sturkenboom M, Hill C, Veldhuyzen van Zanten S, Kuipers EJ. Low-dose acetylsalicylic acid use and the risk of upper gastrointestinal bleeding: a meta-analysis of randomized clinical trials and observational studies. Can J Gastroenterol. 2013;27:159-67.

29. García Rodríquez LA, Gaist D, Morton J, Cookson C, González-Pérez A. Antithrombotic drugs and risk of hemorrhagic stroke in the general population. Neurology. 2013;81:566-74.

30. D'Ascenzo F, Taha S, Moretti C, Omede P, Grossomarra W, et al. Meta-analysis of randomized controlled trials and adjusted observational results of use of clopidogrel, aspirin, and oral anticoagulants in patients undergoing percutaneous coronary intervention. Am J Cardiol. 2015;115:1185-93.

31. Lanas A, Carrera-Lasfuentes P, Arguedas Y, Garcia S, Bujanda L, Calvet X, et al. Risk of upper and lower gastrointestinal bleeding in patients taking nonsteroidal anti-inflammatory drugs, antiplatelet agents, or anticoagulants. Clin Gastroenterol Hepatol. 2015;13:906-12.

32. Bhala N, Emberson J, Merhi A, Abramson S, Arber N, Baron JA, et al. Vascular and upper gastrointestinal effects of non-steroidal anti-inflammatory drugs: meta-analyses of individual participant data from randomised trials. Lancet. 2013;382:769-79.

\section{Submit your next manuscript to BioMed Central and we will help you at every step:}

- We accept pre-submission inquiries

- Our selector tool helps you to find the most relevant journal

- We provide round the clock customer support

- Convenient online submission

- Thorough peer review

- Inclusion in PubMed and all major indexing services

- Maximum visibility for your research

Submit your manuscript at www.biomedcentral.com/submit 